\title{
Concepciones y Prácticas Evaluativas Declaradas por los Docentes en Respuesta a las Necesidades Educativas Especiales de Carácter Permanente
}

\section{Conceptions and Assessment Practices Declared by Teachers as a Response to the Permanent Special Educational Needs}

\author{
Mirtha Jara Henríquez \\ Pilar Jara Coatt * \\ Universidad Católica de la Santísima Concepción, Chile
}

\begin{abstract}
El objetivo de este artículo es dar a conocer las concepciones y prácticas evaluativas de profesores(as) regulares y educadores(as) de Educación Especial, con estudiantes que presentan necesidades educativas especiales, asociadas a discapacidad. La metodología responde a un enfoque cualitativo, de carácter fenomenológico y tipo estudio de caso intrínseco, con la aplicación de entrevistas sobre aspectos del proceso evaluativo y adecuación curricular. El instrumento fue aplicado a 10 participantes: 7 profesores(as) y 3 educadoras de Educación Diferencial de un establecimiento de dependencia administrativa, particular subvencionado. Para el análisis de la información se utilizó el análisis de contenido. El estudio concluye que los participantes realizan adecuaciones curriculares o modificaciones a elementos del currículo para que los estudiantes en situación de discapacidad puedan avanzar en su aprendizaje, sin embargo, las concepciones sobre evaluación y prácticas, mantienen un enfoque tradicional, lo cual demanda un desafío para los centros educativos en implementar propuestas evaluativas flexibles respecto a qué evaluar, cuándo evaluar, cómo evaluar o quién evalúa, reconociendo la particularidad de los(as) estudiantes con o sin necesidades educativas especiales, logrando pleno acceso y participación en el aprendizaje.
\end{abstract}

Descriptores: Educación especial; Integración educativa; Evaluación; Diversificación de la educación; Adaptación escolar.

The aim of this article is to make known the conceptions and evaluative practices of regular teachers and Special Education educators, with students who have special educational needs, associated with disability. The methodology responds to a qualitative approach, of phenomenological character and intrinsic case study type, with the application of interviews about aspects of the evaluation process and curricular adaptation. The instrument was applied to 10 participants: 7 professors (as) and 3 educators of Differential Education of an administrative dependency establishment, private subsidized. For the analysis of the information, the content analysis was used. The study concludes that participants make curricular adjustments or modifications to elements of the curriculum so that students with disabilities can advance their learning, however, conceptions about evaluation and practices, maintain a traditional approach, which demands a challenge for the educational centers to implement flexible evaluative proposals regarding what to evaluate, when to evaluate, how to evaluate or who evaluates, recognizing the particularity of the students with or without special educational needs, achieving full access and participation in learning.

Keywords: Special educational; Educational integration; Evaluation methods; Diversification of education; School adjustment.

*Contacto: pilarjara@ucsc.cl

ISSN: 0718-7378

www.rinace.net/rlei/
Recibido: 24/07/2018

$1^{a}$ Evaluación: 23/07/2018

Aceptado: 20/08/2018 


\section{Introducción}

La educación chilena, dentro de las acciones en la búsqueda de la calidad y equidad, ha incorporado a la Educación Especial dentro de sus niveles educacionales, esta modalidad se encuentra de manera transversal y tiene el propósito de prestar apoyo a los(as) estudiantes según las necesidades específicas que presenten a lo largo de su vida estudiantil. Sin embargo, en estos últimos años, gracias a la promulgación de las leyes y decretos de inclusión educativa como la Ley 20.422/2010 (MIDEPLAN, 2010); Decreto 170/2009 (MINEDUC, 2009a); Decreto 83/2015 (MINEDUC, 2015), se han producido cambios en las prácticas dentro de la sala de clases.

Dichos cambios dejan atrás la antigua pretensión de la Educación Especial como aquella que brindaba apoyos centrados en la búsqueda de medidas remediales, compensatorias y que pretendían nivelar competencias de los(as) estudiantes fuera del aula. Hoy, se busca entregar apoyos a través de una respuesta educativa pertinente para que los(as) estudiantes puedan acceder y participar del proceso de enseñanza y aprendizaje dentro del aula, independiente de las Necesidades Educativas Especiales (en adelante NEE) que presente.

Estos cambios conllevan innovaciones dentro de la comunidad educativa entre las que destacan, la incorporación de distintos profesionales, organizados como equipos interdisciplinarios que funcionan de forma cohesionada, buscando un único fin: dar cuenta de apoyos contextualizados dentro del escenario social y cotidiano de los(as) estudiantes. Entre estos profesionales se encuentran la figura del profesor(a) de diferencial, cuya formación profesional comúnmente obedece a un campo de trabajo diferenciado. Sin embargo, en este nuevo escenario, ha tenido que interiorizarse de aspectos metodológicos y evaluativos que anteriormente no estaban considerados dentro de sus tareas (Godoy, Meza y Salazar, 2004; Torres, 2013). El profesor(a) de asignatura, como otro profesional involucrado, también ha tenido que modificar su rol prototípico al tener que comprender las características de $\operatorname{los}(\mathrm{as})$ estudiantes que presentan NEE y las exigencias que ello conlleva.

Precisamente esta relación bidireccional entre profesor(a) de asignatura y profesor(a) diferencial, suele ser una de las problemáticas derivadas de la nueva visión sobre la inclusión propuesta por las actuales políticas del país a nivel educativo, es decir, acciones de respeto a la diversidad y mayor participación de todos y cada uno de los(as) estudiantes.

Algunas investigaciones sobre las concepciones y actitudes sobre el profesorado respecto de la inclusión educativa (López, Echeita y Martín, 2009) concuerdan en que la integración es un factor crítico para los sistemas educacionales y es una política que debe ser implementada para garantizar el derecho social de acceso y permanencia para el alumnado que presenta dificultades en sus aprendizajes (Romero y Lauretti, 2006). Sin embargo, en un ámbito más específico como la evaluación en el contexto de las NEE permanentes, las investigaciones en Hispanoamérica y Chile son menos numerosas.

Entre las investigaciones sobre concepciones del profesorado respecto de la evaluación en las NEE, Echeita y Calderón (2014) discuten sobre las evaluaciones psicopedagógicas y por extensión, sobre la tarea de la orientación en las instituciones escolares, en particular en el caso del alumnado considerado con necesidades educativas especiales y a la luz del derecho que les asiste a una educación inclusiva. Destacan la necesidad de combatir las concepciones contrarias a la inclusión educativa y hacen un llamado a construir una 
auténtica evaluación inclusiva que ofrezca aportes al sistema educativo en materia de atención a la discapacidad. A su vez, destacan la necesidad de adoptar una perspectiva sistémica que permita mejorar los diferentes elementos del proceso educativo.

En este mismo sentido, San Martín (2012) describe las concepciones que se poseen respecto a los dilemas que supone el diseño de la propuesta curricular y evaluativa para atender a la diversidad del alumnado. Sus hallazgos señalan la presencia de dos perspectivas centrales: modelo clásico de evaluación (teoría directa) y modelo de evaluación continua y formativa (teoría constructiva), reconociendo la necesidad de individualizar la evaluación. Por otro lado, los sujetos investigados manifiestan dificultades para llevar estos planteamientos a la práctica debido a: conflictos para determinar las calificaciones o estudiantes que no presentan NEE. Además, identifican la presencia de teorías implícitas en las que se cree que la realización de evaluaciones diferenciadas se asocia con una mera disminución de la complejidad y número de los ítems.

En cambio, los hallazgos de Damm (2009) dan cuenta que los(as) profesores(as) evidencian en sus prácticas pedagógicas actitudes de indiferencia, sobreprotección, bajas expectativas y aceptación. Las representaciones del profesorado sobre el alumnado con NEE dan cuenta de un imaginario basado en el modelo clínico rehabilitador para abordar las necesidades de aprendizaje de niños y niñas.

Para dar cuenta de la situación en que se encuentra esta problemática se ha realizado una investigación con el fin de indagar sobre la relación entre las concepciones del proceso evaluativo y las prácticas declaradas por profesores(as) de asignatura y profesores(as) de educación diferencial, para responder a las NEE de estudiantes en situación de discapacidad, destacando que los resultados pueden ser la base para innovaciones y propuestas de formación continua, en tanto, aquellos aspectos didácticos, metodológicos o actitudinales podrán ser abordados en investigaciones futuras, considerando el presente trabajo como su precedente.

\section{Revisión de la literatura}

Para abordar los elementos esenciales del estudio en base a investigaciones previas, se desarrollarán los conceptos de Necesidades Educativas Especiales, Concepto de evaluación y prácticas evaluativas desde las concepciones de los(as) docentes.

\subsection{Necesidades Educativas Especiales (NEE)}

Dentro de los sistemas educativos, se han venido haciendo progresivos esfuerzos por incorporar la diversidad dentro de políticas como elemento intrínseco de la dinámica pedagógica. Dentro de esa diversidad resulta crucial considerar a los(as) estudiantes que presentan dificultades de aprendizaje (González González, 2008). En este sentido y desde una perspectiva sociológica, la preocupación por las NEE tiene como meta resguardar el orden y el equilibrio social desde los sistemas educativos (Blanco, 1992). Para ello, resulta importante considerar acciones tendientes a integrar a las personas que no se acomodan a lo que la sociedad requiere como un estándar, por lo que el diagnóstico y la intervención permite disponer de recursos y prever soluciones a modo de ajuste para producir la mencionada (Blanco, 1992).

Surge así la atención a las que se ha denominado como Necesidades Educativas Especiales, término muy utilizado en sistemas escolares como España y Chile. Según el Libro Blanco 
para la Reforma del Sistema Educativo de España, las NEE promueven "la comprensión de la limitación que determinadas condiciones de vida y de escolarización suponen para el desarrollo de las personas" (MEC, 1989, p.163).

El término NEE se incorporaron dentro de las políticas educativas, según López y Valenzuela (2015), a partir de "las conferencias mundiales sobre Educación para Todos realizadas en 1990 y sobre Necesidades Educativas Especiales desarrolladas en 1994, en conjunto con el Marco de Acción Dakar de 2000"(p. 47), que comenzaron a delimitarlas de acuerdo con criterios clínicos asociados a las discapacidades y características psicológicas, sociales y culturales de cada niño(a), que derivaban en dificultades de aprendizaje o de adaptación (UNESCO, 1994). Es un concepto que abarca todo tipo de barreras que un(a) estudiante puede presentar para acceder y participar en su proceso de enseñanza y aprendizaje (MINEDUC, 2009a; López y Valenzuela, 2015).

En conformidad con este marco internacional para la atención a las NEE, las políticas estatales introducen una legislación que aporta al sistema educacional chileno importantes herramientas que garantizará el derecho al acceso de todas las personas a una educación con equidad. El decreto 170/2009, enmarcado en la Ley 20.201, propone una serie disposiciones o normas para el abordaje de las NEE que son beneficiados de subvención para Educación Especial. Dicho decreto concede, desde una perspectiva más bien clínica (López y Valenzuela, 2015), una clasificación de las barreras educativas, las que siguen el siguiente orden:

- Necesidades Educativas Especiales de carácter transitorio: barreras que experimentan ciertos estudiantes en un momento de su vida estudiantil como consecuencia de un trastorno o discapacidad diagnosticada y que requieren ayudas o apoyos adicionales para acceder y participar del currículo por un tiempo determinado. Las NEE de carácter transitorio pueden presentarse asociadas a Dificultades de aprendizaje (DEA), Trastornos Específicos del Lenguaje (TEL), Trastorno por Déficit Atencional (TDA) y Funcionamiento Intelectual Limítrofe (FIL).

- Necesidades Educativas Especiales de carácter permanente: barreras que experimentan ciertos estudiantes durante toda su vida estudiantil como consecuencia de una discapacidad diagnosticada y que requieren ayudas o apoyos extraordinarios para asegurar el acceso y la participación en el currículo. Las NEE de carácter permanente se presentan asociadas a situación de Discapacidad visual, Discapacidad Auditiva, Disfasia, Trastorno Espectro Autista, Discapacidad Intelectual y Discapacidad Múltiple.

Estudios se refieren a la educación inclusiva en el sentido de generar espacios en la escuela a fin de evitar cualquier práctica de exclusión (Hontangas y De la Puente 2010), la narración de las experiencias pedagógicas, el trabajo colaborativo y la meta-reflexión sistemática pueden provocar cambios en la prácticas de aula (Olmos, Romo, Arias, 2016), sin embargo, desde la formación de los docentes se deben incluir las prácticas inclusivas e integradoras que permitan responder a la diversidad de estudiantes en diferentes contextos y que ellos se sientan en las mismas condiciones que sus compañeros (Bustos et al, 2010; Herrera et al, 2018) y avanzar de la teoría a la práctica de educación inclusiva (Fajardo, 2017). 


\subsection{Concepto de evaluación}

El concepto de evaluación ha evolucionado durante los años, usualmente definiciones enfatizan las funciones tradicionales de medición que se le asigna a la evaluación dentro del proceso educativo. Algunos enfoques están centrados en el control y la calificación, mientras que otras, asumen una perspectiva orientadora, formativa y en búsqueda de la mejora, apoyo y orientación de los procesos educativos (Casanova, 2007; Castillo, 2002; González, 2001; Martínez y García, 2008).

Desde la mirada del modelo tradicional, la evaluación suele ser sumativa y no formativa. Su propósito se centra en una valoración al final de un proceso sin la intención de mejorar los aspectos evaluados, puesto que el afán de verificación de este enfoque no permite la retroalimentación (Amaranti, 2010; Pimienta, 2008; Rodríguez y Rivilla, 2013). Asimismo, este enfoque posee un carácter selectivo estándar, ya que establece la posición en que se encuentra un estudiante respecto al grupo curso y no atiende a necesidades individuales. Esta visión de la evaluación con un sentido de control y verificación, comprobación y sanción, clasificación y discriminación se ha centrado en la transmisión de saberes y adquisición de los mismos (Casanova, 2007; Santos, 1995); produciendo una desvinculación o separación del proceso de aprendizaje, reflejando sólo el resultado o procesos concluidos.

Por otro lado, el modelo actual de evaluación aspira al modelo constructivista, centrado en el proceso de aprendizaje y, por lo tanto, promueve una evaluación formativa que da cuenta de una ruta completa y no sólo de la meta. Esta visión actual de la evaluación ha provocado confusiones entre los profesionales de la educación adscritos al enfoque tradicional, debido a la confrontación con la evaluación que se realiza continuamente dentro del aula de tipo sumativa. En este sentido, la evaluación debe dirigirse hacia la mejora de los aprendizajes, en la cual se apoye, oriente y refuerce a los estudiantes, enfatizando hacia la construcción de conocimientos de manera significativa y con sentido (Ahumada, 2005; Capó, Pla y Capó, 2011; Jalo y Pérez, 2016; Moreno, 2011).

Autores se refieren a las interrogantes de la evaluación (Pérez, 2011; Ahumada, 2002; Castro, Correa y Lira, 2006), entre ellas se debieran desarrollar todos los momentos de la evaluación, es decir, inicio, proceso y final con el propósito de diagnóstico, formativo y sumativo respectivamente (Blanco, 1996; Casanova, 2007; Rosales, 2009). Para Fernández-Jiménez, Sánchez y Cabezas (2017) y Santos (2003) la evaluación autoevaluación y coevaluación favorecen los aprendizajes en mayor medida que la heteroevaluación. Por último, la manera en cómo se evalúa debería considerar la gran variedad de procedimientos e instrumentos evaluativos que permiten recoger información válida y confiable de los aprendizajes de los estudiantes, aun cuando hay experiencias en donde no se aprovechan estos resultados para una evaluación que retroalimente de manera efectiva los aprendizajes de los estudiantes (Parrilla, 2010; Salas y Rizo, 2014).

Si nos situamos en un modelo más reciente y que cobra más sentido por la importancia que tiene en el protagonismo del estudiante en el proceso de aprendizaje, podemos citar autores que desarrollan el concepto de evaluación auténtica, es así como Vallejo y Molina (2014) plantean que son instrumentos referidos al criterio y no a la norma, relevando especial importancia cuando se habla de equidad en el reconocimiento del derecho a la diversidad, en tanto, para Álvarez (2005) la evaluación auténtica incide positivamente en la motivación del aprendizaje y valor educativo. Para Muntaner (2009) la evaluación debe ser participativa. Finalmente, este tipo de evaluación favorece la retroalimentación de los 
aprendizajes de los estudiantes entregándoles herramientas para la mejora (Casanova, 2007; Pimineta, 2008; Rosales, 2009).

\section{3. Prácticas evaluativas a partir de sus concepciones}

$\mathrm{El}$ actuar profesional docente corresponde a todos los aspectos que deben ser cubiertos en la labor pedagógica, la cual se ha influenciado de los conocimientos de formación inicial y continua, pero además de la experiencia atesorada durante sus años de ejercicio. Este bagaje va determinando a lo largo de la carrera docente la forma de llevar a cabo, entre muchas otras cosas, el conocimiento del currículum, la planificación de la enseñanza, la reflexión y evaluación sobre su propia práctica, los efectos que tiene su forma de trabajar sobre el grupo de estudiantes (Fernández, 1995; Prieto y Contreras, 2008).

Las concepciones evaluativas permean las acciones de los docentes en los procesos de evaluación (Castillo y Colmenares, 2017), sin embargo, al no existir claridad en dichas concepciones epistemológicas se genera mayor resistencia a los cambios, en especial en la evaluación formativa (García, 2014). La evaluación educativa se ha transformado a lo largo de los años en cuanto a sus funciones y concepciones, originando diversos modelos vinculados a los movimientos sociales de la época (Rodríguez y Rivilla, 2013).

Actualmente, la Educación Especial forma parte de todos los niveles y las modalidades de educación en nuestro país, tanto en los establecimientos de educación parvularia, básica y media cuyo propósito es resguardar a los estudiantes con necesidades educativas especiales, contar con las medidas pedagógicas pertinentes, apoyos y recursos técnicos, humanos y materiales necesarios para progresar en el currículo general en un contexto educativo integrador y lo más inclusivo posible, ya sea de forma temporal o permanente (Díaz, Figueroa, Tenorio, 2008; Espinoza y Valdebenito, 2016; Godoy, Meza y Salazar, 2004; Muñoz, 2018). Por lo tanto, contempla la participación de todos los docentes, asistentes de la educación y comunidad educativa en la implementación de planes acorde a las necesidades educativas especiales con énfasis en el acceso y participación mediante adecuaciones en el currículum que se imparte, el trabajo colaborativo (Francis et al, 2016; Herrera et al., 2018; Martínez y García, 2017) para considerar la evaluación como un proceso valioso y que requiere de un acompañamiento permanente para considerarlo como un enfoque formativo inclusivo.

Al hablar de algunas prácticas inclusivas, Muñoz (2018) plantea considerar a los(as) alumnos(as) en la evaluación de sus compañeros(as) y en su propio aprendizaje, permitiendo que los resultados que se obtienen contribuyan como insumo en la generación de propuestas de mejora. No obstante, aún no existe consenso sobre la inclusión educativa y en las prácticas se ocultan experiencias excluyentes (Guasp, Ramón y Mayo, 2016), por lo tanto, "el profesorado tiene una serie de necesidades formativas que ha de cubrir para responder adecuadamente a la diversidad” (Azorín, 2017, p. 1055).

En relación a lo anterior, los ajustes o medidas de apoyo pueden ser realizados a nivel de acceso al currículum con modificaciones a los materiales, la organización y el personal que rodea al estudiante, así como a nivel de elementos del currículum con ajustes a los objetivos, la metodología, la secuenciación, la temporalización y, la evaluación (MINEDUC, 2015). En este escenario, se entiende, como evaluación diferenciada a todas las adaptaciones en el proceso evaluativo para responder a las necesidades de las y los estudiantes. 
Las adaptaciones que se pueden realizar en el proceso evaluativo buscan responder a las necesidades del estudiante en relación a qué, cómo y cuándo evaluar sus aprendizajes, encontrándose (Aranda, 2002; Duk, Hernández y Sius, 2005; MINEDUC, 2009b):

- Adaptaciones no significativas, las cuales apuntan a la modificar la selección o técnicas e instrumentos de evaluación.

- Adaptaciones significativas, las cuales apuntan a introducir criterios de evaluación específicos, así como modificar los criterios de promoción del estudiante de acuerdo a sus características.

Estas adaptaciones corresponden a recursos o estrategias con que cuenta los(as) docentes para llevar a cabo un desempeño profesional en un grupo que integra estudiantes con necesidades educativas especiales, lo cual exige al docente acercarse a los estudiantes para tener presente los antecedentes y conocimientos sobre las competencias que posee, así como los apoyos que requiere para avanzar en su proceso de aprendizaje, pudiendo implementar actividades de aprendizaje que promuevan la experimentación y el descubrimiento de nuevos esquemas cognitivos. El problema no se encuentra en las características del estudiante sino que en las oportunidades y medios que favorecen su desarrollo (Guasp, Ramón y Mayo, 2016).

\section{Método}

El estudio se enmarcó dentro de un enfoque cualitativo de investigación (Pérez, 2004), de carácter fenomenológico (Gurdián-Fernández, 2007) y con un diseño de caso intrínseco (Stake, 2010), develando las concepciones y prácticas evaluativas de los sujetos participantes del estudio a través de lo que ellos declaran. Las concepciones que tienen los sujetos sobre los fenómenos, influyen directamente en la práctica, por lo tanto, si queremos mejorar el actual docente, es necesario conocer qué es lo que piensan los sujetos desde su comprensión de la realidad (Flick, 2004; Taylor y Bodgan, 2000).

El propósito del estudio fue determinar la relación entre las concepciones del proceso evaluativo y las prácticas declaradas por profesores(as)de asignatura y profesor(a) de educación diferencial, para responder a las Necesidades Educativas Especiales de estudiantes en situación de discapacidad (incluyendo Discapacidad leve, Discapacidad moderada, Trastorno Espectro Autista).

El trabajo se desarrolló a partir de un conjunto de categorías y subcategorías de análisis, generadas a priori, las que surgieron tras la revisión exhaustiva de los aportes teóricos de diversos autores (Ahumada, 2002; Castro, Correa y Lira, 2006; Pérez, 2011). Dichas categorías y subcategorías fueron complementadas con otras de carácter emergentes, las cuales surgieron durante el análisis de los datos, es decir, de lo declarado por los(as) participantes del estudio (cuadro 1).

\section{Participantes}

Los sujetos participantes del estudio corresponden a profesores(as) de asignatura y profesoras de Educación Diferencial de un establecimiento de dependencia administrativa de la comuna de Talcahuano. Quienes accedieron a participar de forma voluntaria. Se consideraron solamente docentes que trabajan con estudiantes que presentan NEE de carácter permanente del Programa de Integración Escolar. 
Cuadro 1. Categorías y subcategorías

El muestreo se realizó de forma intencional, considerando criterios de formación académica en pedagogía, el tipo de necesidad educativa especial con que trabajan los docentes (NEE de carácter permanente) y disponer de horario de trabajo colaborativo.

En total, se incluyeron 10 participantes, a los cuales se le ha asignado un código de acuerdo a la especialidad de cada uno y los años de servicio (por ejemplo: PEM-10, corresponde a Profesor de Enseñanza Media con 10 años de experiencia laboral), para facilitar la representación del contenido de las entrevistas (cuadro 2).

Cuadro 2. Características y docentes participantes

\begin{tabular}{ll}
\hline \multicolumn{1}{c}{ CóDIGO } & \multicolumn{1}{c}{ DOCENTE } \\
\hline PED-O & Profesora Diferencial. Seis meses de experiencia \\
PED-1 & Profesora Diferencial. 1 año de experiencia \\
PED-3 & Profesora Diferencial.3 años de experiencia \\
PEB-1 & Profesora General Básica. 1 año de experiencia \\
PEM-10 & Profesor de Español. 10 años de experiencia. \\
PEB-12 & Profesora General Básica. 12 años de experiencia \\
PEB-13 & Profesora General Básica. 13 años de experiencia \\
PEB-18 & Profesora General Básica. 18 años de experiencia \\
PEM-31 & Profesor de Español. 31 años de experiencia \\
PEB-34 & Profesora General Básica. 34 años de experiencia \\
\hline
\end{tabular}

Fuente: Elaboración propia

\section{Procedimiento de recolección de datos}

Para obtener los datos se utilizó la entrevista (Buendía, Colás y Hernández, 1998) con un guion de preguntas de características semiestructurado. Antes de la aplicación a los participantes, fue enviado a validación con tres académicos que contribuyeron a la corrección y mejora de dicho instrumento. Los(as) docentes corresponden a un Doctor en Educación, Magíster en Ciencias de la Educación y Magíster en Ciencias de la Educación, mención Evaluación. Posterior a ello, se utilizó un aparato de registro sonoro para facilitar 
la construcción de los corpus, de manera tal que al realizar los análisis, no se perdiera ningún dato relevante (cuadro 3).

Cuadro 3. Guion de preguntas

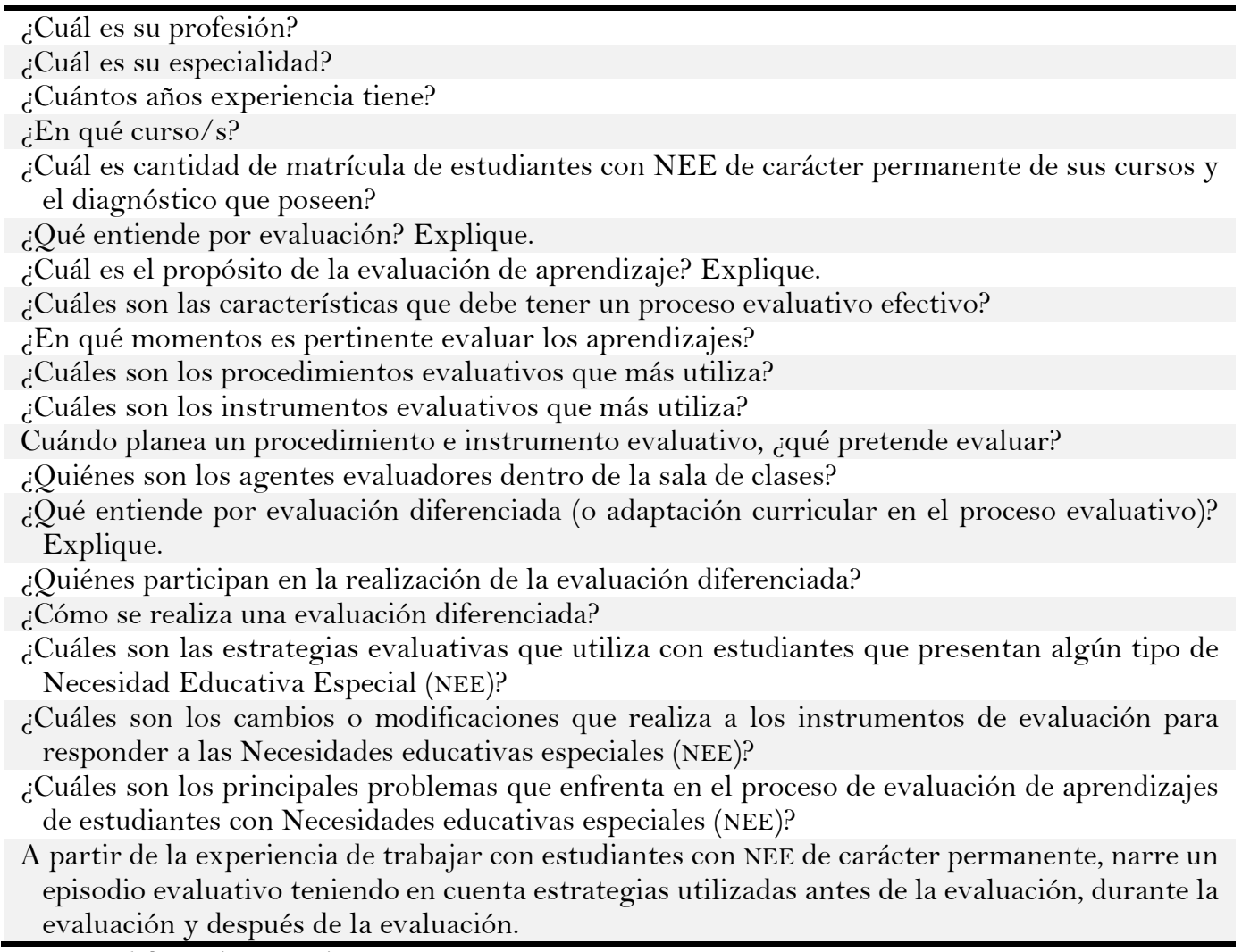

Fuente: Elaboración propia

\section{Procedimientos}

Con la aplicación de las entrevistas, se procede al análisis de las respuestas de los participantes del estudio, recurriendo a la transcripción de manera literal del discurso oral a partir de las grabaciones realizadas. En este estudio, se utilizó el análisis de contenido, fragmentando y recopilando el discurso de los participantes en códigos que permitieron establecer categorías apriorísticas y emergentes, así como sub-categorías. Este análisis permitió reducir, categorizar, clarificar, sintetizar y comparar la información (Pérez, 2011) para obtener los resultados.

\section{Resultados}

A continuación, se presentan algunos resultados obtenidos y sintetizados en las siguientes categorías y sub-categorías:

La categoría nociones sobre evaluación corresponde a las nociones o conocimientos teóricos que los(as) profesores(as) de asignatura y profesoras diferenciales poseen respecto a la evaluación dentro del proceso educativo.

En la sub-categoría concepto de evaluación, los(as) participantes definen la evaluación como un proceso de medición, incluyendo dos ideas dentro de su discurso. Por una parte, 
el aspecto procesual que supone la evaluación, y por otra, la cuantificación de los aprendizajes.

La forma cómo podemos medir el avance de los estudiantes, si se están logrando las cosas que... o los objetivos" (PEM-1O). "Es un proceso que se utiliza para medir conocimiento y aprendizaje. (PED-1)

En la sub-categoría características de la evaluación, las apreciaciones docentes se orientan hacia la congruencia que debe existir entre los objetivos evaluados con los objetivos trabajados en el aula, así como la posibilidad de que la evaluación permita ser aplicada a todos y cada uno de los(as) estudiantes. Los elementos mencionados se complementan con la necesidad de conocer a los(as) estudiantes que se evaluarán, diseñando procedimientos e instrumentos con enunciados claros, manejar una buena redacción y ortografía y poseer dominio del objetivo a evaluar.

Primero, conocer a la persona que voy a evaluar, analizar qué estrategias voy a utilizar también, cuánto saben ellos y cómo voy a abordar el objetivo que quiero evaluar. (PEB-18)

En la sub-categoría momentos de la evaluación, existen participantes que declaran que la evaluación se realiza en todos los momentos de la clase de manera que es posible visualizar los procesos de los estudiantes frente a los objetivos de aprendizaje que se están trabajando, sin embargo, existe una cantidad reducida de participantes que considera a la evaluación como el procedimiento que se desarrolla al final de una unidad u objetivo.

En el inicio, el desarrollo y el cierre... del objetivo en realidad. Es que, en realidad,
eso hablando como de una evaluación formal porque en general uno está evaluando
siempre, incluso con preguntas también uno va al inicio, indaga, aunque sea la clase
va indagando siempre al inicio, ocupa el monitoreo en las actividades para evaluar el
desarrollo y, en los cierres uno también hace evaluaciones que no son formales nada
más. (PEB-1)

La categoría contenidos que evalúan los docentes equivale a aquellos saberes que los docentes consideran al momento de evaluar.

En las subcategorías, se aprecia declaraciones de los(as) participantes tendientes hacia la evaluación principalmente de habilidades (saberes procedimentales) y competencias (saberes conceptuales, procedimentales y actitudinales).

To evalúo habilidades, eso es lo primero que busco según los objetivos de aprendizajes
del curso. (PEB-18)

La categoría agente evaluador de los aprendizajes corresponde a las personas que participan en el proceso de evaluación.

Con respecto a las sub-categorías, se menciona la heteroevaluación dentro de las declaraciones como una de las concepciones que poseen los(as) profesores(as) cuando deben decidir la modalidad evaluativa, priorizando que el docente evalúe a sus estudiantes.

$$
\text { Directamente es la profesora del proyecto y yo. (PEB-34) }
$$

Por otro lado, en las sub-categoría: autoevaluación y coevaluación, algunos(as) docentes expertos mencionan que éstas son modalidades poco utilizada entre las estrategias evaluativas.

Que ellos construyan los instrumentos no, aunque podrían, uno podría dejar un espacio sobretodo en mesa redonda para preparar estas preguntas y ver si las preguntas son pertinentes al tema tratado. To no he trabajado todavía con disertaciones en este curso $2^{\circ}$ medio, pero en cursos superiores sí, de todas maneras. Claro, preguntas, entrevistas, 
por ejemplo, ellos las preparan las entrevistas, son bien hábiles en términos generales. (PEM-3 1)

La categoría prácticas evaluativas declaradas por docentes se relaciona con la acción docente en la implementación de estrategias evaluativas, incluyendo procedimientos, instrumentos e ítems.

En la sub-categoría procedimientos, los(as) profesores(as) tienden a implementar actividades evaluativas basadas en la observación, el desempeño y la interrogación. No obstante, recurren en mayor proporción a procedimientos evaluativos orales y escritos como prácticas habituales con sus estudiantes.

Los más clásicos por así decirlo: las pruebas escritas, las evaluaciones de lectura, los talleres de escritura, diálogos sobre un tema determinado, algunas mesas redondas hacemos. Distintos tipos de evaluación, diversos, pero principalmente la escritura. (PEM-31)

En relación de la sub-categoría instrumento, los participantes hacen alusión al uso de pruebas y guías en la mayoría de las oportunidades evaluativas.

Como lo mencionaba, las pruebas, principalmente, las guías también. (PEB-34)

En la sub-categoría ítems, las apreciaciones reflejan que tienden a utilizar los ítems de selección múltiple y verdadero- falso como práctica recurrente entre los(as) profesores(as) , pese a mencionar otros como términos pareados, completación y respuesta breve.

...también las preguntas de opción múltiple, verdadero y falso, entre otras. (PED-O)

La categoría evaluación diferenciada se relaciona con las estrategias que permiten al docente evaluar el logro de aprendizaje en estudiantes que presentan NEE.

En la sub-categoría propósito de la evaluación diferenciada, se pudo evidenciar que los participantes asumen la evaluación diferenciada como una práctica que efectivamente se desarrolla en el establecimiento educativo y destacan de ella, tres grandes aspectos: responde a las características de los estudiantes con NEE, no es tradicional y estaría determinada por condiciones individuales. Por otro lado, percepciones de algunos participantes conciben a la evaluación diferenciada como la manera de identificar las dificultades de los estudiantes con necesidades educativas especiales. Existen también, aquéllos que consideran como una manera de facilitarse el acceso al proceso evaluativo.

\footnotetext{
Evaluación que es realizada pensando en las características individuales de los estudiantes con necesidades educativas especiales y, en base a los objetivos propuestos a trabajar con ellos" "Tratar de, a lo mejor, sintetizar o hacer las preguntas de manera más precisas, más directa, para que logren entender. (PED-3)
}

En la sub-categoría compromiso con la evaluación diferenciada, los(as) docentes participantes hacen referencia a la comunicación permanente como una forma para responder a las demandas de los(as) estudiantes con NEE, existiendo un grupo profesional especializado, declarando la dedicación por parte de ellos para un trabajo efectivo.

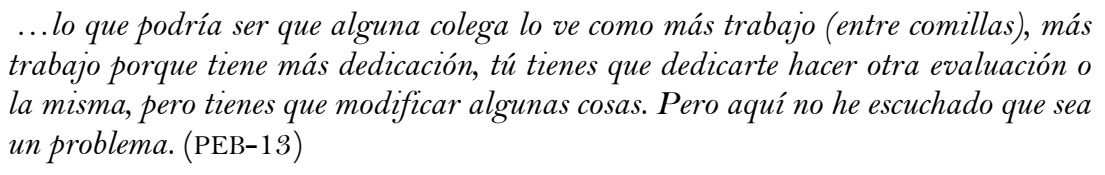
trabajo porque tiene más dedicación, tú tienes que dedicarte hacer otra evaluación o la misma, pero tienes que modificar algunas cosas. Pero aquí no he escuchado que sea un problema. (PEB-13)

En la sub-categoría barreras en la implementación de la evaluación diferenciada, los(as) participantes declaran al momento de responder a las necesidades individuales se presentan barreras debido a la cantidad de estudiantes por curso, la falta de tiempo para 
planear y socializar las estrategias dentro del aula. A la vez, se menciona las características personales de los estudiantes con NEE de carácter permanente, como un impedimento para adecuar los procedimientos evaluativos.

Los principales problemas... y la cantidad de alumnos dentro del aula. Son la
cantidad de curso porque no se puede realizar una evaluación tan minuciosa con cada
niño" (PED-3). "El tiempo. En primer lugar, el tiempo, estamos muy muy limitados
de tiempo. Para hacer una evaluación, se requiere sentarse, analizar y formular bien
las preguntas pensando en ellos. Y el tiempo es muy limitado" (PEB-18). "Con la
incapacidad de saber si el estudiante realmente va a poder hacerla, si se la va a poder
en ese sentido. To creo para mi es lo más complejo, el ver si realmente se la va a poder.
(PEM-10)

La categoría tipos de adecuaciones al proceso evaluativo de estudiantes con NEE de carácter permanente corresponde a los cambios al proceso evaluativo que permita responder a las NEE que presenta un o una estudiante en situación de discapacidad.

En la sub-categoría modificaciones de acceso a la evaluación, los(as) participantes declaran que realizan estrategias para que $\operatorname{los}($ as) estudiantes puedan acceder a la evaluación y participar de mejor forma en ella. Algunas acciones manifestadas fueron: destacar y utilizar enunciados, precisos, breves y claros, distribuir el espacio de mejor forma, disminuir alternativa, eliminar ítems o preguntas, permitir una respuesta oral, uso de recurso concreto, brindar tiempo adicional, aplicar evaluación en etapas, dar explicaciones personalizadas, ubicación estratégica del estudiante, conducción de la respuesta del estudiante , cambio de evaluación, flexibilidad en el procedimiento, reducir la cantidad de calificaciones y contención emocional.

\footnotetext{
Entonces una respuesta explícita no tiene mayor problema, pero cuando yo le hago preguntas implícitas ahi se le presenta una dificultad así que esas las hacemos en forma oral” (PEB-34). “...o quitarle algunos puntos que puedan tener el mismo objetivo, pero quizás acortarle un poco la cantidad de ejercicios. (PEB-12)
}

En la sub-categoría modificaciones a objetivos de aprendizaje, los(as) participantes consideran dentro de las estrategias de respuesta educativa a las adecuaciones a los(as) objetivos de aprendizajes que están desarrollando en el aula según las características de los estudiantes. Algunas acciones señaladas fueron: priorizar uno o más objetivo de aprendizaje, graduar uno o más objetivo de aprendizaje y eliminar uno o más objetivos de aprendizajes.

Cuando hay preguntas demasiado abstractas, evitarlas y hacer preguntas que vaya en relación de lo más concreto; cortar preguntas, si las preguntas tienen tres o cuatro secciones... reducirlas entonces a dos o a una. (PEM-31)

\section{Discusión}

Las apreciaciones expresadas en las entrevistas de $\operatorname{los}($ as) profesores(as) y, respecto a la ejecución de un trabajo pedagógico con conocimientos y estrategias en respuesta a la diversidad, arrojan como resultados de esta investigación, que aún se está en deuda con dichas propuestas (Espinosa y Valdebenito, 2016). Entre algunos elementos, la visón segmentada y parcializada del currículum (Olmos, Romo y Arias, 2016), y en especial, el hecho de considerar la evaluación fuera del proceso de enseñanza- aprendizaje, y no como dentro de éste, ha permitido que se continúe con prácticas de exclusión en los(as) estudiantes. 
Si bien la evaluación la entienden como un proceso inherente al proceso de aprendizaje, en la cual se incorporan a las actividades de la clase para verificar los aprendizajes alcanzados por los(as) estudiantes, no se centra necesariamente en la calificación. A la vez, en la mayoría de docentes prevalece la heteroevaluación como la práctica evaluativa habitual, incluyendo a profesores(as) de asignatura y profesores diferenciales. En este sentido, son ellos quienes los que implementan procedimientos, instrumentos y tipos de reactivos, caracterizados por un enfoque tradicional, orientados al desarrollo de conocimientos y habilidades, según el discurso dado, apartando de sus perspectivas la evaluación de actitudes.

Dicha tendencia hacia estas creencias, consideradas como "tradicionales", trae consigo un distanciamiento del ideal evaluativo que promueve una función formativa y orientadora de la evaluación, la cual contribuye a la toma de decisiones que permita mejorar durante el proceso educativo y, a la vez, permite también la flexibilización al momento de aplicar procedimientos, situaciones e instrumentos evaluativos que reúnan información relevante. Asimismo, se relega de esta forma al estudiante a un rol pasivo dentro de la responsabilidad de administrar su aprendizaje (López e Hinojosa, 2005), aspecto característico del enfoque formativo de la evaluación, donde ya no debería participar sólo el docente (Casanova, 2007) sino cada estudiante asume un rol evaluador de su aprendizaje y enseñanza, donde se respeten sus ritmos y estilos de aprendizaje (Martínez y García, 2017).

Por otro lado, los(as) docentes dentro de sus prácticas declaran haber implementado diversas estrategias evaluativas hacia estudiantes en situación de discapacidad, las cuales permitirían acceder al procedimiento o instrumento confeccionado para el curso general, sin embargo, todos(as) los(as) estudiantes con y sin NEE tienen características particulares que le diferencian de los demás (González, 1998). Por lo tanto, el desafío de la evaluación es evidenciar dificultades, logros, desempeños y hechos relevantes, relacionado con el desempeño del estudiante y del profesor.

Entre las medidas para responder a las NEE asociadas a situación de discapacidad, se destacan aquellas que favorecen el acceso y la participación dentro del currículo, como: destacar instrucciones, usar enunciados precisos, otorgar tiempo adicional, permitir responder oralmente, conducir la respuesta, entre otras. Y, en algunos casos, se ajusta el objetivo de aprendizaje a las competencias del estudiante. Sin embargo, parecería que la estrategia evaluativa que declaran practicar los profesores, no conceden un lugar especial o primordial a la atención a la diversidad. Esto podría ser mirado desde una perspectiva de equidad, puesto que no se haría diferencias entre estudiantes a la hora se ser evaluados en sus aprendizajes, es decir, todos los(as) alumnos(as) serían iguales en este sentido. Una mirada distinta tendería a ver esta medida como un aspecto segregador, puesto que los(as) estudiantes en situación de discapacidad tenderían a estar en una situación de desventaja frente a sus compañeros(as) dadas sus necesidades particulares.

En relación a lo anterior, se aprecia un limitado repertorio de estrategias utilizadas en la evaluación de aprendizajes, tanto en la variedad de procedimientos como de instrumentos, a pesar que los profesores reconocen los distintos estilos, ritmos, motivaciones, intereses y características para aprender, especialmente si presenta NEE de carácter permanentes (MINEDUC, 2017). Este desfase entre lo que declaran conocer y aplicar respecto de las estrategias evaluativas, podría deberse a las limitaciones naturales que el contexto educativo presenta: alto número de estudiantes dentro de la sala de clases, falta de tiempo 
para aplicar estrategias personalizadas o dedicación de largos periodos a la regulación del clima de aula.

\section{Conclusiones}

El propósito de esta investigación fue analizar la relación entre las concepciones y prácticas evaluativas implementadas por profesores(as) de asignatura y profesoras(es) diferencial, para responder a las Necesidades Educativas Especiales de estudiantes en situación de discapacidad, pudiendo concluir que desde el punto de vista tradicional, aun se siguen manteniendo ciertas prácticas: los profesores conciben la evaluación como medición, evaluando solo los objetivos de los programas, con una heteroevaluación primordialmente y con procedimientos, instrumentos e ítem de alternativas y respuesta alterna.

Por otra parte, respecto de los principios de la evaluación auténtica, solo a nivel declarativo plantean que en sus prácticas la evaluación está presente en todo momento, que el propósito de la evaluación diferenciada es atender a las características individuales de $\operatorname{los}$ (as) estudiantes, que mantienen un compromiso con la evaluación diferenciada, haciendo uso de enunciados más precisos, disminuir la cantidad de alternativas en pruebas escritas, incluir respuestas orales, reducción de calificaciones, entre otras actividades.

Entendemos que la clasificación dada por los resultados de la evaluación de la calidad de la educación en Chile, en las escuelas y profesores(as), no permiten avanzar a la igualdad (Ferrada, 2017), siendo una señal poderosa desde las políticas educativas, no obstante, una solución para esta dicotomía sería que los(as) profesores(as) tuvieran a disposición una mayor variedad de estrategias para atender a esta diversidad dentro del aula, de modo que no se comprometa la igualdad de condiciones que debería propiciar el desarrollo del proceso educativo, es decir, profesionales más especializados en la formación en educación inclusiva (Ruiz-Bernardo, 2016), más aun cuando las barreras para la implementación de la evaluación diferenciada, desde el discurso de los(as) profesores(as), es la cantidad de estudiantes, la falta de tiempo para planear una mayor cantidad de estrategias evaluativas, por lo tanto, es necesario tomar decisiones desde la política pública.

El abordaje de las NEE demanda un desafío de los(as) profesores(as) y centros educativos, quienes al poseer concepciones que promuevan la atención a la diversidad conlleva prácticas pedagógicas hacia el reconocimiento de las particularidades en sus estudiantes, favoreciendo así el acceso y la participación en su aprendizaje, logrando su desarrollo personal y social. Lo mencionado, en muchos casos, requiere la incorporación de adecuaciones curriculares a nivel individual respecto a qué enseñar, cómo enseñar, en cuándo enseñar, qué evaluar o cómo evaluar, lo cual impulsa un proceso reflexivo y flexible para unificar criterios para responder a sus necesidades educativas, asimismo, demanda que $\operatorname{los}(\mathrm{as})$ docentes y profesionales involucrados en el proceso de enseñanza-aprendizaje, trabajen de manera colaborativa y como un equipo cohesionado e interdisciplinario que planifique, ejecute y evalúe acciones implementadas hacia los(as) estudiantes.

Si bien existe normativa que establece una clasificación de NEE asociados a un diagnóstico en particular y que requiere capacitación de parte del profesorado, no se debe desconocer que dentro del aula se encuentran estudiantes que, por sus condiciones personales, sociales o académicas, presentan dificultades para avanzar respecto al currículo de su curso, quienes, con apoyos profesionales, técnicos o materiales, acceden a una educación no 
excluyente sino inclusiva con prácticas pedagógicas hacia altas expectativas y aceptación a todos y cada uno de sus estudiantes.

Nuestro sistema educativo se encuentra camino hacia la inclusión, evidenciando acciones con los(as) estudiantes con NEE asociados a discapacidad y, son los(as) docentes quienes participan en este proceso de acogida y comprensión de la diversidad con iniciativas de formación o capacitación continua, colaboración y capacidad para crear situaciones educativas cercanas y pertinentes al alumnado.

\section{Referencias}

Ahumada, P. (2002). La evaluación en una concepción de aprendizaje significativo. Valparaíso: Ediciones Universitarias de Valparaíso.

Ahumada, P. (2005). Hacia una evaluación auténtica del aprendizaje. Ciudad de México: Paidós.

Álvarez, I. (2005). Evaluación como situación de aprendizaje o evaluación auténtica. Perspectiva Educacional, Formación de Profesores, 45, 45-68.

Amaranti, M. (2010, mayo). Evaluación de la educación: Concepciones y prácticas de retroalimentación de los profesores de lenguaje y comunicación de primer año de educación media investigación cualitativa con estudio de caso. Comunicación presentada en el Congreso Iberoamericano de Educación, Metas 2021. Universidad de Buenos Aires, Argentina.

Aranda, R. (2002). Educación especial. Madrid: Pearson Educación S.A.

Azorín, C. (2017). Análisis de instrumentos sobre educación inclusiva y atención a la diversidad. Revista Complutense de Educación, 28(4), 143-160. https://doi.org/10.5209/RCED.51343

Blanco, A. (1992). Educación especial y sociología. Revista Interuniversitaria de Formación del Profesorado, 13, 201-208.

Blanco, L. (1996). La evaluación educativa, mas proceso que producto. Lleida: Edicions Universitat de Lleida.

Buendía, L., Colás, P. y Hernández, F. (1998). Métodos de investigación en psicopedagogía. Madrid: Mc Graw-Hill.

Bustos, L., Ramírez, N., Molina, M., Jenny, C. y Sandra, S. (2010). Formador de formadores ante los retos de una educación inclusiva. Praxis Pedagógica, 10(11), 90-99. https://doi.org/10.26620/uniminuto.praxis.10.11.2010.90-99

Capó, J., Pla, C. y Capó, J. (2011). La evaluación como elemento de mejora y enriquecimiento del sistema educativo. Revista Latinoamericana de Estudios Educativos, 41, 17-34.

Casanova, M. (2007). Manual de evaluación educativa. Madrid: La Muralla.

Castillo, N. y Colmenares, A. (2017). Contrato pedagógico: Imaginario social en la práctica democrática evaluativa. Praxis Pedagógica, 17(20), 39-62. https://doi.org/10.26620/uniminuto.praxis.17.20.2017.39-62

Castillo, S. (2002). Compromisos de la evaluación educativa. Madrid: Pearson Educación S.A.

Castro, F., Correa, M. y Lira, H. (2006). Curriculum y evaluación educacional. Santiago: Ediciones Universidad del Biobío.

Damm, X. (2009). Representaciones y actitudes del profesorado frente a la integración de niños/as con necesidades educativas especiales al aula común. Revista Latinoamericana de Educación Inclusiva, 3, 25-35. 
Díaz, T., Figueroa, A. y Tenorio, S. (2008). Las competencias del educador diferencial en problemas de aprendizaje en el contexto de implementación del decreto 291. Contextos, Estudios de Humanidades y Ciencias Sociales, 20, 65-73.

Duk, C., Hernández, A. M. y Sius, P. (2005). Las adaptaciones curriculares: Una estrategia de individualización de la enseñanza. Disponible en http://www.mistalentos.cl/userfiles/files/Adap\%20Curr\%20Cynthia\%2oDuk.pdf

Echeita, G. y Calderón, I. (2014). Obstáculos a la inclusión: Cuestionando concepciones y prácticas sobre la evaluación psicopedagógica. Àmbits de Psicopedagogia i Orientació, 41, 67-98.

Espinosa, J. y Valdebenito, V. (2016). Explorar las concepciones de los docentes respecto al proceso de educación inclusiva para la mejora institucional. Revista Latinoamericana de Educación Inclusiva, 1O(1), 195-213. https://doi.org/10.4067/So718-73782016000100010

Fajardo, M. (2017). La educación superior inclusiva en algunos países de Latinoamérica: Avances, obstáculos y retos. Revista Latinoamericana de Educación Inclusiva, 11(1), 171-197. https://dx.doi.org/10.4067/S07 18-73782017000100011

Fernández, M. (1995). La profesionalización docente. Perfeccionamiento. Investigación en el aula. Análisis de la práctica. Madrid: Siglo XXI.

Fernández-Jiménez, C., Sánchez, M. y Cabezas, M. (2017). Aplicación de la autoevaluación en una experiencia de aprendizaje basado en problemas con alumnado de educación en asignaturas relacionadas con la discapacidad. Estudios Sobre Educación, 32, 73-93. https://doi.org/10.15581/004.32.73-93

Ferrada, D. (2017). Identidad docente frente a la calidad como estandarización en las escuelas de la región del Biobío. Revista Latinoamericana de Educación Inclusiva, 11(1), 93-107. https://doi.org/10.4067/So718-73782017000100007

Flick, U (2004). Introducción a la investigación cualitativa. Madrid: Ediciones Morata

Francis, L., Gross, J., Blue-Banning, M., Haines, S. y Turnbull, A. (2016). Directores escolares y padres que logran resultados óptimos: Lecciones aprendidas de seis escuelas norteamericanas que han implementado prácticas inclusivas. Revista Latinoamericana de Educación Inclusiva, 1O(1), 43-60. https://dx.doi.org/10.4067/S07 18-73782016000100004

García, L. (2014). Evaluación formativa de los aprendizajes en el contexto universitario: Resistencias y paradojas del profesorado. Educación XX1, 17(2), 35-55.

Godoy, P., Meza, M. y Salazar, A. (2004). Antecedentes históricos, presente y futuro de la educación especial en Chile. Santiago: Ministerio de Educación.

González, M. (1998). Necesidades educativas especiales/generales hacia una nueva denominación. Revista Galego-Portuguesa de Psicoloxía e Educación, 2(3), 89-94.

González, M. (2001). La evaluación del aprendizaje: Tendencias y reflexión crítica. Revista Cubana de Educación Superior, 15(1), 85-96.

González González, M. T. (2008). Diversidad e inclusión educativa: algunas reflexiones sobre el liderazgo en el centro escolar. REICE. Revista Iberoamericana sobre Calidad, Eficacia y Cambio en Educación, 6(2), 82-99.

Guasp, J., Ramón, M. y Mayol, B. (2016). Buenas prácticas en educación inclusiva. Educatio Siglo XXI, 34(1), 31-49. https://doi.org/10.6018/j/252521

Gurdián-Fernández, A. (2007). El paradigma cualitativo en la investigación socio-educativa. San José de Costa Rica: IDER. 
Herrera, J., Parrilla, A., Blanco, A. y Guevara, G. (2018). La formación de docentes para la educación inclusiva. Un reto desde la universidad nacional de educación en Ecuador. Revista Latinoamericana de Educación Inclusiva, 12(1), 21-38. https://doi.org/10.4067/So71873782018000100021

Hontangas, N. y De la Puente, J. (2010). Atención a la diversidad y desarrollo de procesos educativos inclusivos. Prisma Social, 4, 1-37.

Jalo, M. y Pérez, M. C. (2016). La evaluación como situación de aprendizaje: El diseño de pruebas auténticas. Puertas Abiertas, 12, 1-19.

López, B. e Hinojosa, K. (2005). Evaluación del aprendizaje: Alternativas y nuevos desarrollos. Ciudad de México: TRILLAS.

López, I. y Valenzuela, G. (2015). Niños y adolescentes con necesidades educativas. Revista Médica Clínica las Condes, 26(1), 42-51. https://doi.org/10.1016/j.rmclc.2015.02.004

López, M. Echeita, G. y Martín, E. (2009). Concepciones sobre el proceso de inclusión educativa de alumnos con discapacidad intelectual en la educación secundaria obligatoria. Cultura y Educación, 21(4), 485-496. https://doi.org/10.1174/113564009790002391

Martínez, B. y García, M. (2008). La formación de competencias docentes para incorporar estrategias adaptativas en el aula. Revista Complutense de Educación, 19(2), 253-274.

Martínez, C. y García, I. (2017). Análisis de las prácticas evaluativas inclusivas de tres docentes de educación preescolar en México. Revista Latinoamericana de Educación Inclusiva, 1 1(1), 225243. https://doi.org/10.4067/S0718-73782017000100014

MINEDUC. (2009a). Guías de adecuaciones curriculares para estudiantes con necesidades educativas especiales. Ciudad de Guatemala: MINEDUC.

MINEDUC. (2009b). Decreto Supremo 170. Fija las normas para determinar los alumnos con necesidades educativas especiales que serán beneficiados de las subvenciones para educación especial. Santiago: MINEDUC.

MINEDUC. (2015). Decreto 83. Aprueba criterios y orientaciones de adecuación curricular para estudiantes con necesidades educativas especiales de educación parvularia y educación básica. Santiago: MINEDUC.

MINEDUC. (2017). Orientaciones sobre estrategias diversificadas de enseñanza para educación básica, en el marco del Decreto 83/2015. Santiago: MINEDUC.

MIDEPLAN. (2010). Ley 20.422, sobre igualdad de oportunidades e inclusión social de personas con discapacidad. Santiago: MINEDUC.

Moreno, T. (2011). La cultura de la evaluación y la mejora de la escuela. Perfiles Educativos, 33(131), 116-130.

Muntaner, J. (2009). Escuela y discapacidad intelectual. Sevilla: Eduforma.

Muñoz, C. (2018). Prácticas pedagógicas en el proceso de transición hacia la escuela inclusiva. Seis experiencias en El Salvador. Revista Latinoamericana de Educación Inclusiva, 12(1), 95-110. https://doi.org/10.4067/S0718-73782018000100095

Olmos, A., Romo, M. y Arias, L. (2016). Reflexiones docentes sobre inclusión educativa: Relatos de experiencia pedagógica sobre la diversidad universitaria. Revista Latinoamericana de Educación Inclusiva, 1O(1), 229-243. https://doi.org/10.4067/So718-73782016000100012

Parrilla, M. (2010). Ética para una investigación inclusiva. Revista de Educación Inclusiva, 3(1), 165174.

Pérez, G. (2004), Investigación cualitativa. Retos e interrogantes. I Métodos. Madrid: La Muralla. 
Pérez, G. (2011). Investigación cualitativa. Retos e Interrogantes. II Técnicas y análisis de datos. Madrid: La Muralla.

Pimienta, J. (2008). Evaluación de aprendizajes. Un enfoque basado en competencias. Ciudad de México: Pearson Educación de México.

Prieto, M. y Contreras, G. (2008). Las concepciones que orientan las prácticas evaluativas de los profesores: Un problema a develar. Estudios Pedagógicos, 34(2), 245-262. https://dx.doi.org/10.4067/S07 18-07052008000200015

Rodríguez, P. y Rivilla, A. (2013). La formación del profesorado en la competencia evaluadora: Un camino hacia la calidad educativa. Enseñanza \& Teaching, 31(2), 167-188.

Romero, R. y Lauretti, P. (2006). Integración educativa de las personas con discapacidad en Latinoamérica. Educere, 10(33), 347-356.

Rosales, C. (2009). Criterios para una evaluación formativa. Sevilla: Narcea S.A. Ediciones

Ruiz-Bernardo, P. (2016). Percepciones de docentes y padres sobre la educación inclusiva y las barreras para su implementación en Lima, Perú. Revista Latinoamericana de Educación Inclusiva, 10(2), 115-133. https://doi.org/10.4067/So7 18-73782016000200008

Salas, A. y Rizo, F. (2014). Evidencias de prácticas de evaluación de un grupo de profesores de primarias de Nuevo León. Revista Mexicana de Investigación Educativa, 19(61), 537-567.

San Martín, C. (2012). Atención de la diversidad en el contexto educativo chileno: Concepciones del profesorado sobre evaluación y diseño de la propuesta curricular. REICE. Revista Iberoamericana sobre Calidad, Eficacia y Cambio en Educación, 10(4), 164-183.

Santos, M. (1995). La evaluación: Un proceso de diálogo, comprensión y mejora. Málaga: Aljibe.

Santos, M. (2003). Una flecha en la diana. La evaluación como aprendizaje. Madrid: Narcea.

Stake, R. (2010). Investigación con estudio de casos. Madrid: Morata.

Taylor, S. y Bodgan, R. (1990). Introducción a los métodos cualitativos de investigación. La búsqueda de significados. Barcelona: Ediciones Paidós.

Torres, A. (2013). Representaciones sociales sobre el proceso evaluación desde la mirada de docentes de $1^{\circ}$ año básico en establecimientos municipalizados urbanos de la comuna de Quilpué. Estudios Pedagógicos, 39(1), 285-304. https://doi.org/10.4067/So71807052013000100017

UNESCO. (1994). Declaración de Salamanca y marco de acción sobre necesidades educativas especiales. Madrid: UNESCO.

Vallejo, M. y Molina, M. (2014). La evaluación auténtica de los procesos evaluativos. Revista Iberoamericana de Educación, 64, 11-25.

\section{Breve CV de las autoras}

\section{Mirtha Jara Henríquez}

Profesora de Educación Diferencial mención Lenguaje, Dificultad de Aprendizaje y Discapacidad Intelectual, Universidad de Concepción. Educadora de Párvulos y Licenciada en Educación, Universidad Católica de la Santísima Concepción. Magister en Ciencias de la Educación, mención Evaluación de los Aprendizajes, de la Facultad de Educación, Universidad Católica de la Santísima Concepción. Chile. Es asesora técnica en temas de inclusión y necesidades educativas especiales en Consultora educativa Bilmark Ltda. ORCID ID: https://orcid.org/OOOO-OOO2-2723-4558. Email:mirthjara@gmail.com 


\section{Pilar Jara Coatt}

Profesora de Educación General Básica y Magister en Ciencias de la Educación, mención Evaluación Curricular, Universidad Católica de la Santísima Concepción. Cursa doctorado en Educación, Universidad Internacional Iberoamericana de México. Línea de investigación: Concepciones y prácticas evaluativas, Innovación y emprendimiento en educación. Investigadora del Centro INNOVAPEDIA ${ }^{\circledR}$ UCSC en proyectos relacionados con la innovación y emprendimiento en educación: FIC 2014-2016, EMPT 2017, PAEI 2017, FIC- R 2018-2020, IIP 2018-2019. Actualmente se desempeña como académica del Departamento de Curriculum y Evaluación de la Facultad de Educación de la Universidad Católica de la Santísima Concepción. Chile, en el desarrollo de docencia de pre y post grado, gestión, vinculación con establecimientos educacionales e investigación. ORCID ID: https://orcid.org/0000-0002-9975-8713. Email: pilarjara@ucsc.cl 